\title{
Studying the impact of sex on the molecular mechanisms of liver adaptation to fasting in mice
}

\author{
N. Bazhan ${ }^{1,2 *}$, N. Sitnikova ${ }^{2}$, T. Iakovleva ${ }^{1}$, A. Dubinina ${ }^{1}$, E. Makarova ${ }^{1}$ \\ ${ }^{1}$ Institute of Cytology and Genetics SB RAS, Novosibirsk, Russia \\ ${ }^{2}$ Novosibirsk State University, Novosibirsk, Russia \\ *e-mail: bazhan-nm@yandex.ru
}

Key words: carbohydrate-lipid metabolism, fasting-refeeding, liver, gene expressions

Motivation and Aim: Fundamental aspects of metabolic homeostasis are known to be regulated differently in males and females. A key adaptive response to fasting is the shift from glucose to lipid oxidation. The liver plays a crucial role in mobilizing energy during nutritional deprivation [1]. It remains unclear if there are sex-dependent peculiarities in molecular mechanisms of liver adjustment to fasting. The aim of this study was to evaluate the expression patterns of key genes involved in lipid and glucose metabolism in liver and their relationship with blood parameters in response to food restriction.

Methods and Algorithms: Male and female C57Bl mice were studied under different feeding conditions: feeding state and $24 \mathrm{~h}$ fasting. Blood parameters and the expression of genes involved in glucose metabolism (gluconeogenesis, glycolysis) and lipid metabolism (lipogenesis, fatty acid oxidation) in liver were analyzed. mRNA levels were measured by RT-PCR.

Results: As an effect of fasting, there was decrease in circulating glucose and leptin levels, increase in FGF21 and adiponectin levels both in male and female mice. There were no differences between male and female mice in fasting induced changes in expression of genes involved in glucose metabolism: mRNA levels of genes controlling gluconeogenesis ( $P c k 1, G 6 P, P g c 1)$ were increased, and glycolysis $(G k)$ was decreased compared to those in fed animals. Transcriptional regulation of hepatic lipid metabolism was sex-dependent. Expression of Ppar- $\alpha$ gene, which is involved in fatty acid oxidation and concomitant production of ketone bodies, was higher in females compare to males both in fed and fasting states. Fasting decreased the expression of lipogenic gene (Fas), and increased the expression of genes related with fatty acid oxidation (Ppar- $\alpha, F g f 21$ Cptl) in mice of both sexes, but fasting induced increase in the Fgf2land Cpt1 gene expressions were more pronounced in female than in male mice.

Conclusion: Thus, in mouse liver, the effect of fasting on the expression of genes involved in glucose metabolism was independent on sex, and on genes involved in fat metabolism was dependent on sex. Increased fatty acid oxidation favors the production of ketone bodies - the main energy source under fasting conditions. Increased expression of hepatic $\mathrm{Fgf} 2 \mathrm{l}$ and $\mathrm{Cpt} 1$ genes in fasted females possibly reflects a higher ability for the female mice to respond to increased energy demands.

Acknowledgements: Supported by the Russian Science Foundation, grant No. 17-1501036.

\section{References}

1. Potthoff M.J. et al. (2009) FGF21 induces PGC-1alpha and regulates carbohydrate and fatty acid metabolism during the adaptive starvation response. Proc Natl Acad Sci USA. 106(26):10853-10858. DOI 10.1073/pnas.0904187106. 